\title{
Inhibition of CXCR4-CXCL12 chemotaxis in melanoma by AMD11070
}

\author{
G O’Boyle ${ }^{1}$, I Swidenbank ${ }^{1,5}$, H Marshall $^{1}$, CE Barker ${ }^{1}$, J Armstrong ${ }^{2}$, S A White ${ }^{1}$, S P Fricker ${ }^{3}$, R Plummer ${ }^{4,6}$, \\ M Wright ${ }^{1,6}$ and PE Lovat ${ }^{\star, 1,6}$ \\ ${ }^{1}$ Institute of Cellular Medicine, Newcastle University, Newcastle upon Tyne NE2 4HH, UK; ${ }^{2}$ University of Sunderland, Sunderland, \\ UK; ${ }^{3}$ Genzyme Corporation, Framingham, MA 01701, USA and ${ }^{4}$ Northern Institute for Cancer Research, Newcastle University, \\ Newcastle upon Tyne NE2 4HH, UK
}

Background: Despite intensive research and novel adjuvant therapies, there is currently no cure for metastatic melanoma. The chemokine receptor CXCR4 controls metastasis to sites such as the liver; however, the therapeutic blockade with the existing agents has proven difficult.

Methods: AMD11070, a novel orally bioavailable inhibitor of CXCR4, was tested for its ability to inhibit the migration of melanoma cells compared with the commonly described antagonist AMD3100.

Results: AMD11070 abrogated melanoma cell migration and was significantly more effective than AMD3100. Importantly for the clinical context, the expression of B-RAF-V600E did not the affect the sensitivity of AMD11070.

Conclusion: Liver-resident myofibroblasts excrete CXCL12, which is able to promote the migration of CXCR4-expressing tumour cells from the blood into the liver. Blockade of this axis by AMD11070 thus represents a novel therapeutic strategy for both B-RAF wild-type and mutated melanomas.

Cutaneous melanoma represents an increasing world health problem. In the United Kingdom alone, melanoma incidence has quadrupled over the past 30 years, making it now the eighth most common malignancy, resulting in more than 2000 deaths a year from malignant disease (Cancer Research UK). Alarmingly, melanoma is now the second most common cancer in the 15-34 years age group, emphasising the critical importance of early intervention to prevent premature loss of life.

While the prognosis for early-stage disease is favourable, being largely treatable through surgical excision alone and resulting in 5-year survival rates of $>95 \%$, the outlook for patients with advanced-stage disease, however, is extremely poor. Despite intensive research, there is currently still no cure for metastatic disease, which commonly targets the liver and results in 5-year survival rates of $<12 \%$ (Thompson, 2012), thus emphasising the acute need for novel treatment strategies and targeted therapies.
Study of genetic factors predisposing to melanoma has led to the discovery of oncogenic mutations in NRAS and B-RAF (Davies, 2002; Dong et al, 2003; de Snoo and Hayward, 2005), which result in constitutive activation of RAF-extracellular signal-regulated kinase $(\mathrm{ERK}) /$ mitogen-activated protein kinase signalling and the promotion of melanoma proliferation and chemoresistance (Gray-Schopfer et al, 2007; Balmanno and Cook, 2009). Although mutated B-RAF has been validated in recent clinical studies as a therapeutic target for melanoma (Smalley, 2010), not all patients harbour activating B-RAF mutations or respond to B-RAF inhibitors, and the emergence of acquired resistance is already recognised as a significant problem in the treatment of B-RAFV600E-mutated disease (Chapman, 2011). An acute demand for alternative personalised approaches for more effective treatment stratification hence remains.

Recently, substantial evidence has shown that chemokines and chemokine receptors, which were first shown to induce directional

\footnotetext{
${ }^{\star}$ Correspondence: Dr P Lovat; E-mail: penny.lovat@ncl.ac.uk

${ }^{5}$ These authors contributed equally to this work.

${ }^{6}$ Joint senior authors.
} 
migration of leukocytes from the blood into organs, may also be involved in cancer metastasis. Chemokines are a family of small chemoattractant cytokine-like proteins $(8-11 \mathrm{kDa})$ that bind to and activate G-protein-coupled seven-transmembrane domain (GPCRs 7TM) receptors. At present, approximately 50 chemokines and at least 20 corresponding receptors have been identified. Classification of the chemokines and their receptors is based on the position and the number of conserved cysteine residues present, and to date, four families have been identified: CXC, CX3C, CC and C. It has been shown that they play an important role in tumour development in a manner similar to leukocyte trafficking by guiding the directional migration of cancer cells towards a gradient of chemotactic cytokines secreted by organs such as the liver. These observations suggest that targeting chemokine receptors may provide a novel therapeutic strategy for preventing metastasis in a wide range of cancers including melanoma.

The most common chemokine receptor known to be expressed by cancer cells is CXCR4. Previous studies have also demonstrated increased expression of CXCR4 in uveal melanoma tumours, which correlated with disease progression (Franco et al, 2010). However, in primary cutaneous melanoma, recent studies suggest that there is a significant correlation between overexpression of CXCR4 and survival or prognosis (Kuhnelt-Leddihn et al, 2012), although this study did not examine differences between primary and metastatic lesions.

The constitutive expression of the CXCR4 ligand, CXCL12, in tissues such as the liver is thought to have a pivotal role in the organspecific dissemination of metastatic disease. It is widely accepted that the expression of chemokine receptors by cancer cells leads to migration, enhanced proliferation and cell survival by a mechanism that includes CXCL12-mediated MAPK signalling (Balmanno and Cook, 2009; Teicher and Fricker, 2010). The CXCR4-CXL12 axis therefore represents an attractive therapeutic target for melanoma (Teicher and Fricker, 2010). However, clinical translation has proven difficult because of poor pharmacological profile of AMD3100, a commonly described antagonist. AMD11070 has recently been described as a novel orally bioavailable inhibitor of CXCR4 with a more favourable pharmacokinetic profile (Mosi et al, 2012); therefore; the aim of this study was to define its potential to inhibit CXCR4-CXCL12-mediated chemotaxis in melanoma.

\section{MATERIALS AND METHODS}

Cell culture and reagents. CHL-1 (B-RAF-wild type (WT)) and A375 (B-RAF-V600E mutated) were cultured in Dulbecco's modified Eagle's medium (DMEM) with $10 \%\left(\mathrm{wv}^{-1}\right)$ fetal calf serum and $1 \%$ glutamine. Cells were maintained at $37^{\circ} \mathrm{C}$ in a humidified $5 \% \mathrm{CO}_{2}$ atmosphere. AMD11070 and AMD3100 (supplied by Genzyme Corporation, Framingham, MA, USA) were freshly prepared at $1 \mathrm{mg} \mathrm{ml}^{-1}$ in citric acid/normal saline solution and diluted to given concentrations in DMEM. Concentrations of $6.6 \mu \mathrm{M}$ AMD11070 or $2 \mu \mathrm{M}$ AMD3100 were typically incorporated in functional assays representing the maximum possible clinically achievable concentrations in vivo allowing comparison of both inhibitors (Stone et al, 2007). Human T cells were isolated and activated as described previously (Newton et al, 2009). CXCL12neutralising antibody (R\&D Systems, Abingdon, UK; Clone 79014) was used at $100 \mu \mathrm{g} \mathrm{ml}^{-1}$.

Immunofluorescence confocal microscopy. Archival human liver tissue from patients with metastatic melanoma was obtained with full ethical approval (Newcastle and North Tyneside Research Ethics Service ref.: 08/H0906/95). Formalin-fixed paraffin-embedded (FFPE) tissue sections were processed for immunofluorescene based on previously described methodology (Hong et al, 2009).
Specifically, FFPE sections were dewaxed in xylene for $10 \mathrm{~min}$ and rehydrated through 100, 95 and $70 \%$ ethanol to running water and washed in TBS ( $\mathrm{pH}$ 7.6; Tris-buffered saline). Antigen retrieval was then carried out by pressure cooking slides in $10 \mathrm{~mm}$ Tris buffer ( $\mathrm{pH}$ 7.4) for $1 \mathrm{~min}$, followed by rapid cooling in running water and washing in TBS. Sections were then blocked with $20 \%$ normal swine serum for $1 \mathrm{~h}$ before incubating with mouse antiCXCR4 antibody $\left(10 \mu \mathrm{g} / \mathrm{ml}^{-1}\right.$, overnight at $4{ }^{\circ} \mathrm{C}$; R\&D Systems; MAB172) or mouse monoclonal antibody to $\alpha$-smooth muscle actin ( $\alpha$-SMA; Sigma-Aldrich, Dorset, UK). Sections were then washed three times in TBS (as between all steps) and incubated with FITC-conjugated goat-anti-mouse IgG (1/100, $1 \mathrm{~h}$; Sigma). Mouse on mouse reagent (Vector Laboratories, Peterborough, UK) was then used to block mouse IgG as per the manufacturer's instructions and sections were blocked again with $20 \%$ normal swine serum for $30 \mathrm{~min}$. Sections were then incubated with the second primary antibody, mouse-anti-MelanA (1/500;1 h, RT; Abcam, Cambridge, UK) and then TRITC-conjugated goat-antimouse IgG (1/100, 1 h; Sigma). Sections were then counterstained with DAPI for $15 \mathrm{~min}$ and autofluorescence blocked using Sudan Black $\left(0.3 \%\left(\mathrm{wv}^{-1}\right)\right.$ in $100 \%$ ethanol; $\left.30 \mathrm{~min}\right)$. Finally, sections were mounted using fluorescence mounting medium (Dako, Stockport, UK).

Myofibroblasts (passage 3) from histologically normal resected liver tissue with informed donor consent and ethical approval from the Newcastle and North Tyneside Research Ethics Committee were cultured as described previously (Haughton et al, 2006). Quiescent cells were cultured on plastic in media containing serum, which then transdifferentiated into a myofibroblast phenotype. Following permeabilisation with methanol and fixation with $2 \%$ formaldehyde/0.2\% glutaraldehyde in PBS, $\mathrm{pH}$ 7.4, fixed cells were incubated in the presence or absence of a primary mouse monoclonal antibody to $\alpha$-SMA (Sigma; $1: 1000$ ) and detected with a secondary anti-mouse IgG (whole molecule) - fluorescein antibody (green) or with an anti-human/mouse CXCL12/SDF-1 mouse monoclonal antibody (R\&D Systems; $1: 1000)$ detected with a secondary anti-mouse IgG (whole molecule)-rhodamine (Sigma; 1:400) antibody (red). Mouse IgG1 isotype primary antibody (Dako; 1:1000) was used a control. Cells were counterstained with DAPI and images were captured by confocal microscopy (original magnification, $\times 20$ ).

Flow cytometry. Analysis of CXCR4 expression was performed as described previously (Mellor et al, 2007) using anti-CXCR4 FITC (MAB172; R\&D Systems). Data were generated using a BD Canto II (Oxford, UK) and analysed using the FlowJo software (Ashland, OR, USA).

Cell viability. The commercial Cell Titer 96 Aqueous NonRadioactive Cell Proliferation Assay (MTS) was used to determine cell viability following 24-h incubation with $6.6 \mu \mathrm{M}$ AMD11070 according to the manufacturer's instructions (Promega, Southampton, UK).

Chemotaxis. Migration assays were carried out as described previously (Mellor et al, 2007) using an $8-\mu \mathrm{m}$ transwell filter (BD, Franklin Lakes, NJ, USA). A total of 200000 cells were placed in the upper chamber in a volume of $500 \mu \mathrm{l}$ with the lower chamber containing $800 \mu \mathrm{l}$ serum-free media supplemented with chemokine. The assay was incubated for $16 \mathrm{~h}$ at $37^{\circ} \mathrm{C}$ before the removal of excess cells and medium from both chambers. The upper surface of the filter was gently swabbed before fixation in $100 \%$ ice-cold methanol for $1 \mathrm{~h}$. Each filter was then stained using haematoxylin before dehydration and mounting. Assays were performed in triplicate, with the migrant cells in nine high-power fields per filter being counted blindly. T cells' isolation and migration were performed as described previously (O'Boyle et al, 2012). 
Western blotting and transfection. Expression vectors for B-RAFWT and B-RAF-V600E (provided by R Marais, Patterson Institute of Cancer Research, Manchester, UK), or pcDNA4 (Invitrogen, Carlsbad, CA, USA) were transiently transfected using Lipofectamine 2000 according to the manufacturer's instructions. Preparation of whole-cell lysates and western blotting for B-RAF (F-7; Santa Cruz Biotechnology, Santa Cruz, CA, USA; diluted 1:1000), ERK1/ 2, phosphorylated (Thr202/Tyr204) ERK1/2 (Cell Signalling Technology, Danvers, MA, USA; diluted $1: 2000$ ) and $\beta$-actin (Sigma; diluted $1: 40000$ ) were performed as described previously (Lovat et al, 2008).

Statistical analyses. All results are expressed as mean values \pm s.e.m. of replicate samples. The significance of changes was assessed by the application of an ANOVA with Bonferroni posttest. All data were analysed using the Prism 5 software (GraphPad, San Diego, CA, USA).

\section{RESULTS}

A series of experiments were performed to demonstrate the rationale underlying this study. A clear physical association between metastatic melanoma tumour cells and liver-resident myofibroblasts was observed in liver biopsy sections from patients with metastatic melanoma (Figure 1A). Strong expression of CXCL12 ligand was observed in primary myofibroblasts (Figure 1B). Costaining of five patients indicated that the metastatic melanoma cells in the liver of these patients express the CXCL12 receptor, CXCR4 (Figure 1C).

Flow cytometry was used to measure the expression of CXCR4 on two commonly used melanoma cell lines (Figure 2A). Both CHL-1 and A375 cells express comparable amounts of the CXCL12 receptor. These cells were migrated in a dose-response assay towards CXCL12, with $10 \mathrm{~nm}$ inducing optimum migration of both cell lines (Figure 2B).

As our study intended to address the effect of the CXCR4 inhibitor AMD11070 on cell migration, it was next necessary to determine if the antagonist inhibitors had a detrimental effect on cell viability. No significant difference in cell viability was observed following culture of A375 or CHL-1 melanoma cells in the presence of increasing concentrations of AMD11070 (Figure 3). The specificity of the inhibitor was then demonstrated using activated T cells. These cells express both CXCR3 and CXCR4 (O'Boyle et al, 2012). AMD11070 had no effect on migration towards the CXCR3 ligand CXCL11, but did inhibit the response to CXCL12 (Figure 4).

AMD3100 and AMD11070 were contrasted for their inhibition of melanoma cell migration. Both inhibitors significantly blocked chemotaxis of CHL-1 cells towards CXCL12 (Figure 5A). In contrast, AMD11070 was more effective at inhibiting migration of A375 than AMD3100 (78\% and 21\% inhibition, respectively, $P<0.05)$. Intriguingly, A375 cells harbouring the B-RAF-V600E mutation displayed higher background migration in the absence of chemokine. A scratch assay was used to assess the effect of the CXCR4 inhibitors (Figure 5B). Cells treated with AMD11070 had a significantly increased void size at all time points assessed $(P<0.05$; Figure 5C).

We next considered the role of B-RAF in melanoma migration towards CXCR4 and sensitivity to the inhibitor AMD11070. Western blotting analysis indicated that both A375 and CHL-1 cells express similar levels of B-RAF overall (Figure 6A), so the increased chemotaxis of A375 cells was not solely due to an overexpression of B-RAF. We subsequently transfected CHL-1 cells with vectors encoding WT and V600E B-RAF (Figure 6B). As expected, this increased the amount of ERK activation in these cells. A migration assay was then performed contrasting mocktransfected with CHL-1 cells overexpressing WT and V600E (Figure 6C). Intriguingly, overexpression of V600E but not WT B-RAF increased the background migration of CHL-1 $(P<0.05)$. Importantly for the therapy of metastatic melanoma, however, overexpression of either form of B-RAF did not affect the capacity of AMD11070 to inhibit migration $(P<0.01)$.

To reflect the recruitment to the liver in vivo, conditioned media were collected from activated primary myofibroblasts. An ELISA was performed to measure CXCL12; media conditioned for $72 \mathrm{~h}$ contained $87 \pm 13 \mathrm{ng} \mathrm{ml}^{-1}$ CXCL12. This media were then used as the stimulus in a chemotaxis assay (Figure 7). As the conditioned

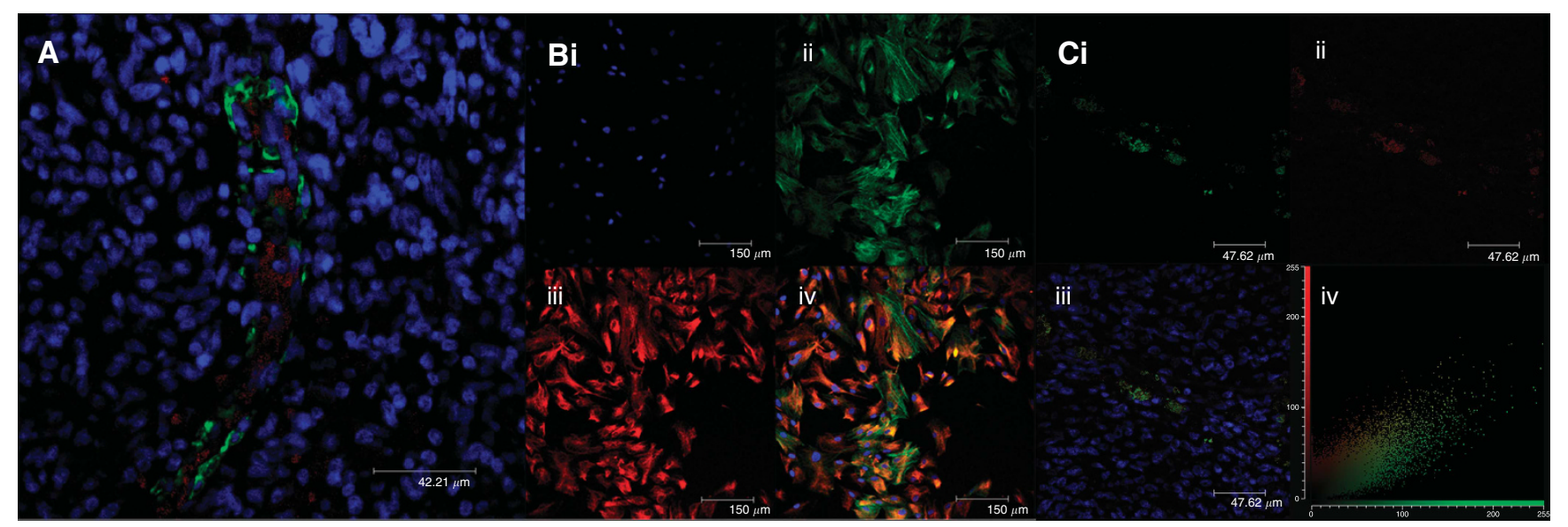

Figure 1. The CXCR4/CXCL12 axis in melanoma migration to the liver. (A) Representative example of immunofluorescence staining of human liver tissue from a patient with melanoma metastasis stained for $\alpha$-SMA (green) and MelanA (red). Original magnification, $\times 20 ;$ scale bar $=42 \mu \mathrm{M}$. Image is representative of five patients. (B) Representative example of immunofluorescence staining of primary myofibroblasts (passage 3). Cells were incubated (i) without primary antibody or (ii) with antibody to $\alpha$-SMA (green) or (iii) with anti-CXCL12 (red). (iv) Overlay of each image with yellow staining indicating costaining of $\alpha$-SMA and CXCL12. Cells were counterstained with DAPI (4',6-diamidino-2-phenylindole) to visualise the nuclei and images were taken by confocal microscopy (original magnification, $\times 20$; scale bar $=150 \mu \mathrm{M}$ ). Image is representative of three patients. (C) Representative example of double immunofluorescence staining of metastatic melanoma sections demonstrating staining with (i) antibody to CXCR4 (green) or (ii) anti-MelanA (red). (iii) Overlay of each image with yellow staining indicating costaining of MelanA and CXCR4. (iv) Cytofluorogram scatterplot of CXCR4 and MelanA staining. Cells were counterstained with DAPI to visualise the nuclei and images were taken by confocal microscopy (original magnification, $\times 20$; scale bar $=150 \mu \mathrm{m}$ ). Image is representative of five patients. 
media may contain several chemotactic factors, an important control experiment was performed using a CXCL12-neutralising antibody to indicate that the importance of the CXCL12-CXCR4

A
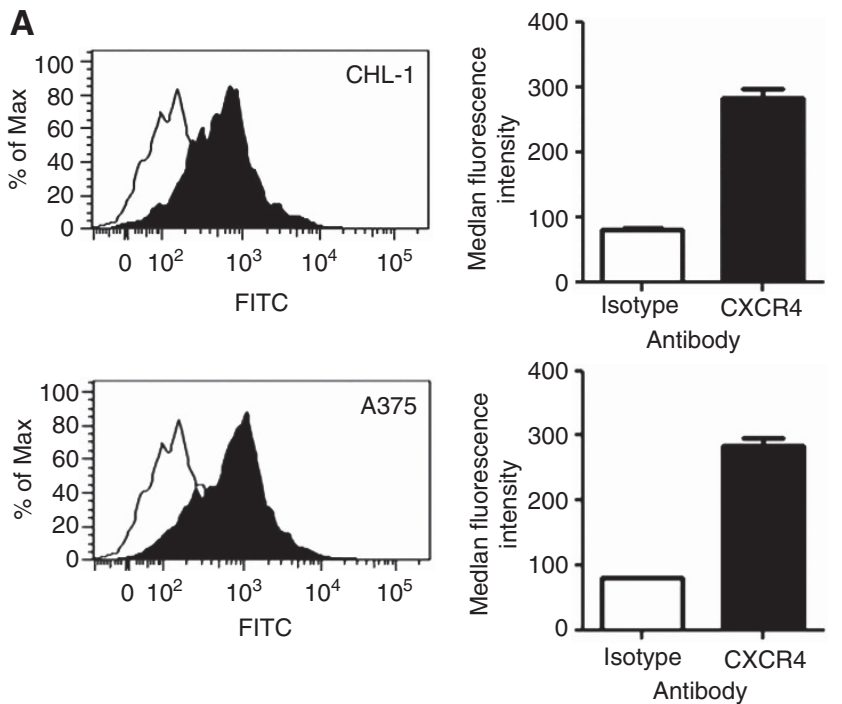

B

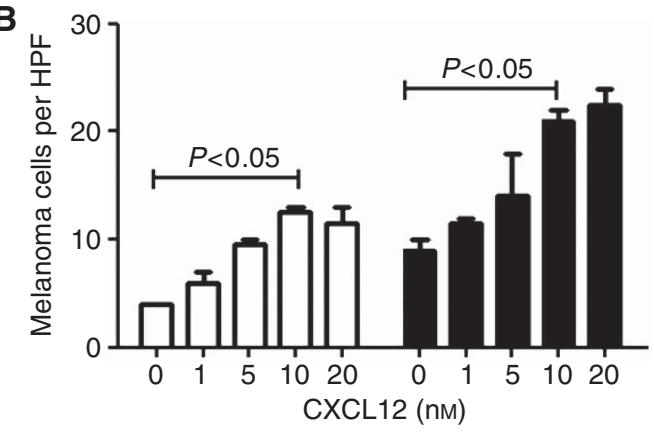

Figure 2. Melanoma cells migrate towards CXCL12. (A) Fluorescenceactivated cell sorting analysis of the expression of the chemokine receptor CXCR4 on two melanoma cell lines: CHL-1 and A375. Black histograms correspond to cells incubated with the CXCR4 receptorspecific antibody, and white histograms correspond to cells incubated with the isotype-matched control antibody. (B) Dose-response migration of melanoma cells line CHL-1 (white bars) and A375 (black bars) migrated for $16 \mathrm{~h}$ across an $8-\mu \mathrm{m}$ pore size filter towards the indicated concentration of CXCL12. Data represent mean values of three independent experiments; error bars correspond to s.e.m. HPF, high-power field. axis; the migration of melanoma cell lines was blocked by CXCL12 neutralisation. The migration response of CHL-1 cells was significantly inhibited by both AMD3100 and AMD11070 $(P<0.05)$; however, only AMD11070 inhibited migration of A375 in this assay $(P<0.05)$.

\section{DISCUSSION}

Chemokine receptors are cell surface G-protein-coupled receptors and therefore amenable to inhibition by small-molecule antagonists. AMD3100 (known as Plerixafor and Mozobil) is a CXCR4 antagonist approved for use to mobilise haematopoietic stem cells to the peripheral blood for collection and subsequent autologous transplantation in patients with non-Hodgkin's lymphoma and multiple myeloma (Calandra et al, 2010). AMD3100 also has demonstrable efficacy in murine models of metastasis (Yasumoto et al, 2006) serving as proof-of-concept for targeting this axis in the management of melanoma. However, the pharmacokinetics of AMD3100 are unsuitable for use in human trials for melanoma therapy. To achieve a therapeutically effective chemokine receptor blockade that limits pathological cell migration, around $90 \%$ receptor occupancy must be achieved with an antagonist (Schall and Proudfoot, 2011).

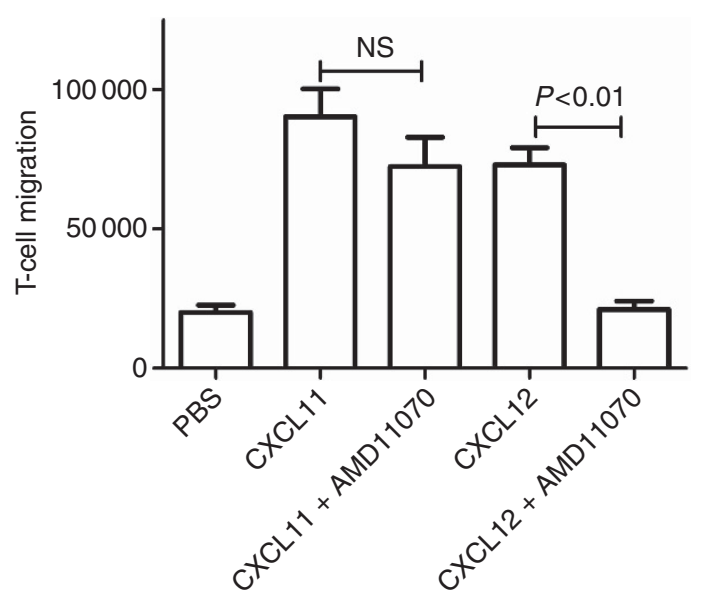

Figure 4. AMD11070 is a specific CXCR4 inhibitor. Absolute migration of activated T cells towards $10 \mathrm{~nm}$ of CXCL11 or CXCL12 with and without $6.6 \mu \mathrm{m}$ AMD11070 for $90 \mathrm{~min}$ across a $3 \mu \mathrm{m}$ pore filter. Data represent mean values of three independent experiments; error bars correspond to s.e.m. NS, not significant; PBS, phosphate-buffered saline.

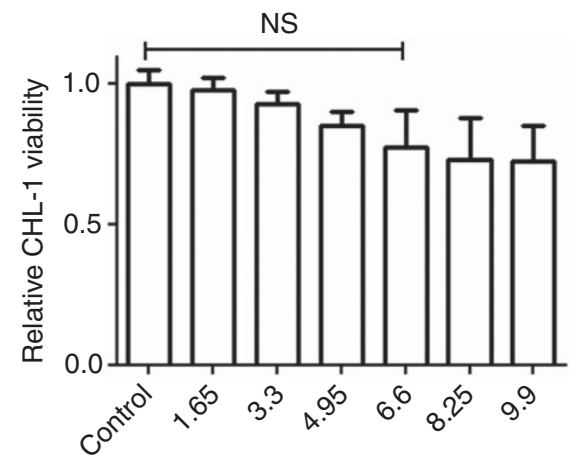

AMD11070 concentrations $(\mu \mathrm{M})$

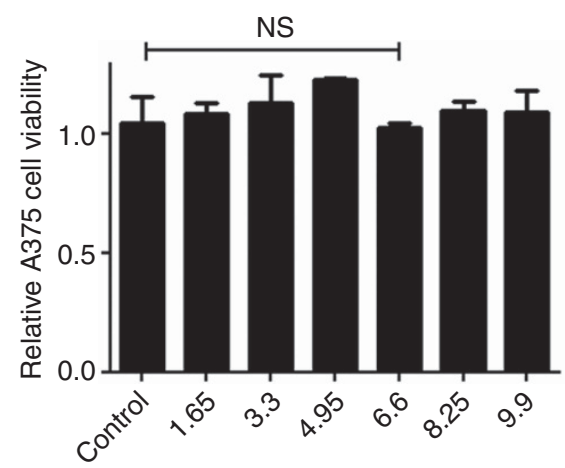

AMD11070 concentrations $(\mu \mathrm{M})$

Figure 3. AMD11070 has no affect on melanoma viability. Cell viability assay following $24 \mathrm{~h}$ treatment of CHL-1 (white bars) and A375 (black bars) with AMD11070. Cell viability was calculated as a percentage relative to vehicle cells. Data represent mean values of three independent experiments; error bars correspond to s.e.m. NS, not signficant. 
A

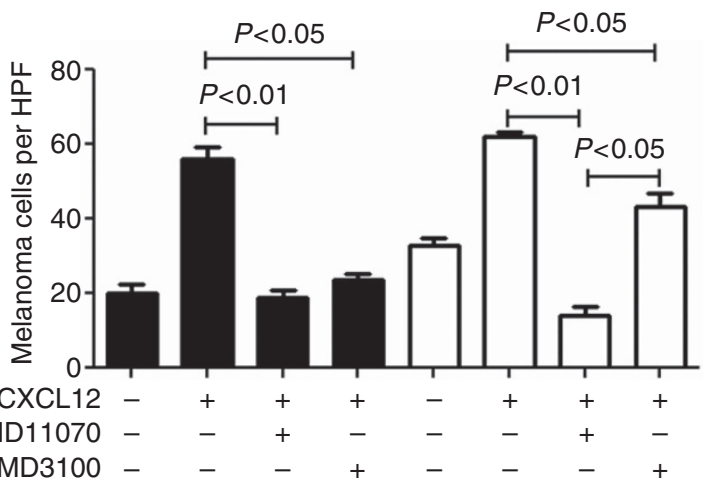

B
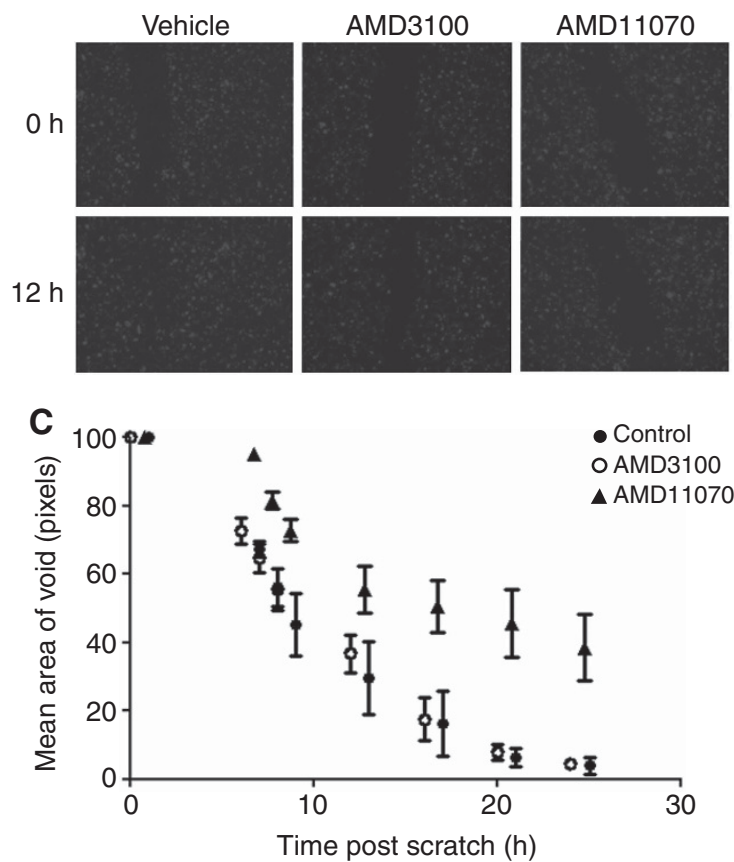

Figure 5. (A) Effect of CXCR4 inhibition on melanoma migration. Chemotaxis assay using CHL-1 (black bars) and A375 (white bars) migrated for $16 \mathrm{~h}$ towards $10 \mathrm{~nm} \mathrm{CXCL12}$ across an $8-\mu \mathrm{m}$ pore size filter in the presence of $6.6 \mu \mathrm{M}$ AMD11070. (B) Live cell scratch assays were performed over $24 \mathrm{~h}$ using $\mathrm{A} 375$ cells stably expressing red fluorescent protein. A375 cells were treated in the presence or absence of either AMD3100 or AMD11070 at their maximum clinically achievable concentrations, with images being captured every 30 min over a $24-h$ period following the creation of an area of void at time 0 . (C) Mean area of void (pixel number) of control A375-RFP cells or following treatment over $24 \mathrm{~h}$ (time $0,6,7,8,12,16,20$ and $24 \mathrm{~h}$ with $2 \mu \mathrm{m}$ AMD3100 or $6.6 \mu \mathrm{M}$ AMD11070. Data represent mean values of three independent experiments; error bars correspond to s.e.m. HPF, high-power field.

AMD11070 is an orally bioavailable antagonist of CXCR4, able to achieve plasma concentrations of $6.6 \mu \mathrm{M}$ (when administered at an oral dose of $400 \mathrm{mg} \mathrm{kg}^{-1}$ in fasted healthy volunteers; Stone et al, 2007), and which selectively inhibits the binding of CXCR4 to CXCL12 (Mosi et al, 2012). No apparent acute toxicity was observed in oral bioavailability studies using AMD11070 and other CXCR4 antagonists have not resulted in any mechanism-based toxicity (Dale et al, 2012), suggesting that CXCR4 is a safe therapeutic target. We therefore examined the ability of AMD11070 to disrupt the chemokine-driven migration of melanoma cells.

Oncogenic mutations in oncogenic B-RAF are common in melanoma, specifically a V600E mutation. Feedback loops in melanoma signalling networks demonstrate that B-RAF-V600E is able to regulate both the MAPK and AKT pathways (Chen et al, 2012).
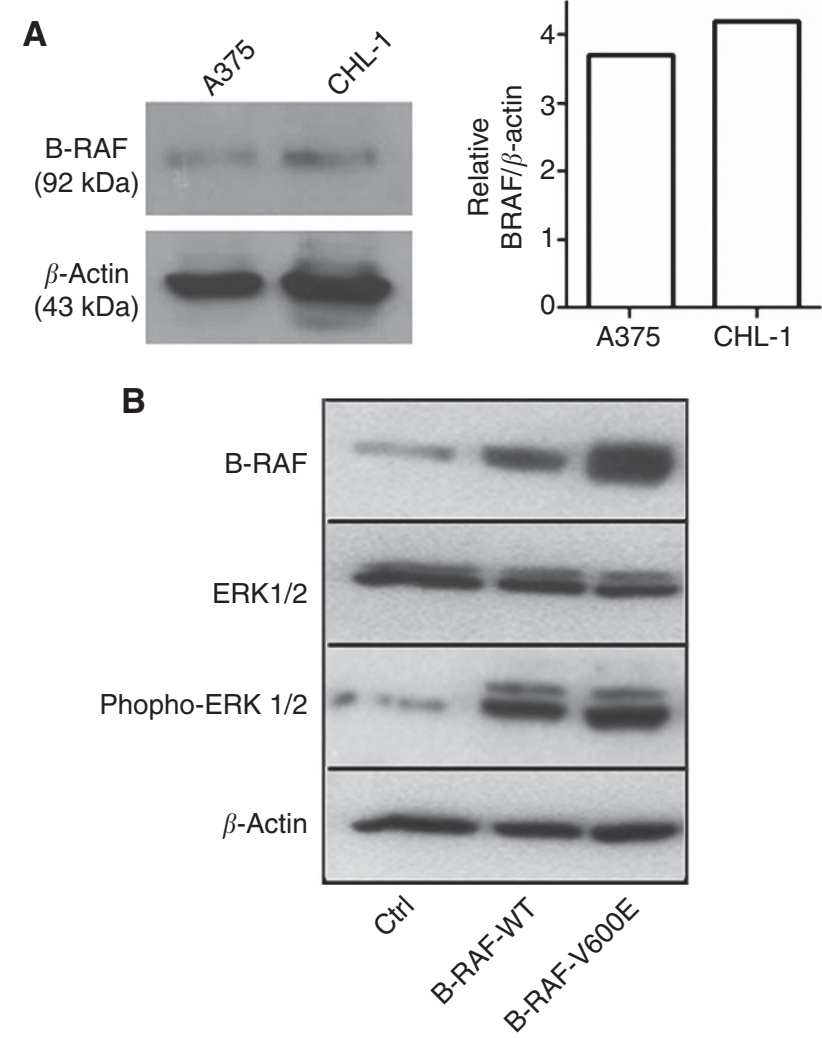

C

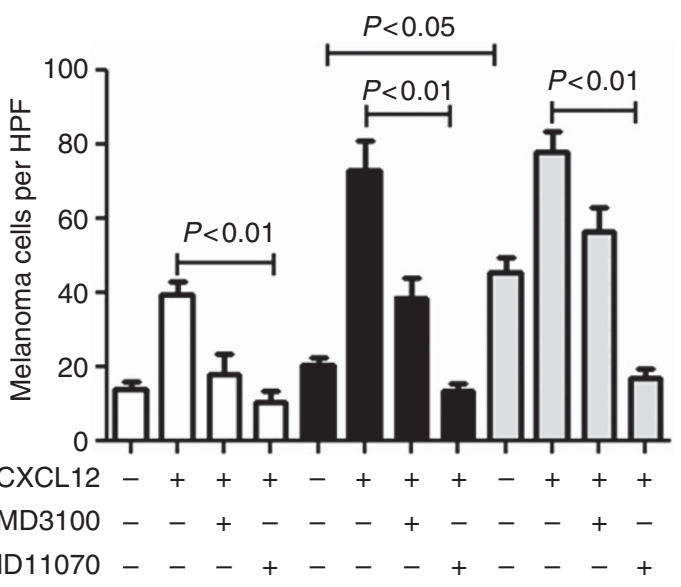

Figure 6. (A) Effect of B-RAF-V600E on melanoma migration. Western blotting for B-RAF in melanoma cell lines with densitometric quantification. (B) Western blotting as indicated in $\mathrm{CHL}-1$ transiently transfected with WT or V600E B-RAF. (C) CHL-1 cells transfected with either WT (black bars) or mutant (V600E) (grey bars) B-RAF migrated for $16 \mathrm{~h}$ towards $10 \mathrm{~nm}$ CXCL12 across an $8-\mu \mathrm{m}$ pore size filter in the presence of $6.6 \mu \mathrm{m}$ AMD11070. HPF, high-power field.

As well as effects on growth and survival, B-RAF may also influence tumour migration. Previous work has demonstrated that B-RAF mediates the migratory response to the chemokine CXCL8 in neutrophils (Knall et al, 1995). CHL-1 cells, which express WT B-RAF were transfected with vectors encoding WT or V600E $\mathrm{B}-\mathrm{RAF}$ and migrated towards CXCL12 in the presence or absence of AMD11070. Consistent with previous studies, transfection of V600E into WT B-RAF melanoma cells increased ERK activation (Chen et al, 2012). Importantly for melanoma, where up to $60 \%$ of patients harbour activating B-RAF mutations, V600E expression did not affect susceptibility to AMD11070. Densitometric analysis confirmed that the vast majority of B-RAF present in these cells 


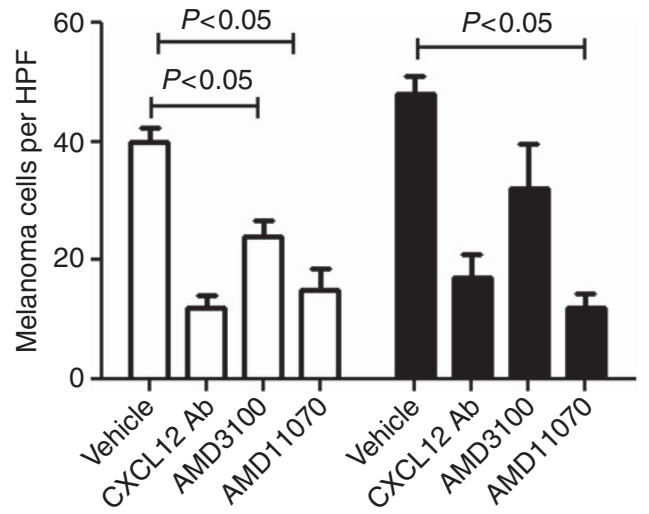

Figure 7. Effect of B-RAF-V600E on melanoma migration. Chemotaxis assay using CHL-1 (white bars) and A375 (black bars) migrated for $16 \mathrm{~h}$ towards conditioned media from activated myofibroblasts across an 8- $\mu \mathrm{m}$ pore size filter in the presence of $100 \mu \mathrm{g} \mathrm{ml}^{-1} \mathrm{CXCL12-}$ neutralising antibody (Ab), $2 \mu \mathrm{M}$ AMD3100 or $6.6 \mu \mathrm{m}$ AMD11070. HPF, high-power field.

was due to the transfected plasmid, and intriguingly, transfection with WT or V600E B-RAF increased the maximum response to CXCL12, suggesting that the total amount of B-RAF is able to modulate chemotaxis. Furthermore, V600E-transfected CHL-1 cells had a significantly higher background migration than mock-transfected or those transfected with WT B-RAF. This suggests a possible additional oncogenic role for $\mathrm{V} 600 \mathrm{E}$, in that these cells were more motile even in the absence of exogenous ligand. However, a confounding caveat is that V600E was expressed at a slightly higher level than WT, a typical observation in this system (Armstrong et al, 2011).

We describe a clear mechanism for the site-specific metastasis of melanoma; liver-resident myofibroblasts excrete CXCL12, which is able to promote the migration of CXCR4-expressing tumour cells from the blood into the liver. Data suggest that AMD11070 will possess antimetastatic therapeutic efficacy in patients with tumours harbouring WT or mutant B-RAF in excess of that achievable with AMD3100 by preventing migration towards condition media from activated primary myofibroblasts. Although there is a potential for other cytokines produced by myofibroblasts to promote tumour metastasis, this experiment indicates that the production of CXCL12 plays a major role in melanoma metastasis, suggesting that antagonism of CXCR4 by AMD11070 will prevent the migration of melanoma cells to the liver. Interestingly, overexpression of mutant B-RAF increased melanoma cell migration but did not abrogate sensitivity to the AMD3100 inhibitor.

Taken together, these data suggest that AMD11070 may provide a promising new therapy for preventing melanoma metastasis to the liver. However, additional preclinical studies are required to solidify our findings and to translate them into a clinical setting. Such efforts would require the validation of a suitable model of human metastatic melanoma, lack of which represents a significant barrier to translational research in metastatic disease. Nevertheless, the benefits of AMD11070 may not be limited only to melanoma patients as CXCR4 is highly expressed by many other cancers, including breast cancer, lung cancer, neuroblastoma, colorectal cancer and ovarian cancer.

\section{ACKNOWLEDGEMENTS}

This work was supported by the British Skin Foundation, The North Eastern Skin Research Fund, Newcastle Healthcare Charity, BBSRC, Arthritis Research UK and The Wellcome Trust (Grant number: WT086755MA). SPF is an employee of Genzyme: a Sanofi company, original developer of AMD3100 and AMD11070.

\section{REFERENCES}

Armstrong JL, Corazzari M, Martin S, Pagliarini V, Falasca L, Hill DS, Ellis N, AL Sabah S, Redfern CP, Fimia GM, Piacentini M, Lovat PE (2011) Oncogenic B-RAF signaling in melanoma impairs the therapeutic advantage of autophagy inhibition. Clin Cancer Res 17: 2216-2226.

Balmanno K, Cook SJ (2009) Tumour cell survival signalling by the ERK1/2 pathway. Cell Death Differ 16: 368-377.

Calandra G, Bridger G, Fricker SP (2010) CXCR4 in clinical hematology. Curr Top Microbiol Immunol 341: 173-191.

Chapman PB, Hauschild A, Robert C, Haanen JB, Ascierto P, Larkin J, Dummer R, Garbe C, Testori A, Maio M, Hogg D, Lorigan P, Lebbe C, Jouary T, Schadendorf D, Ribas A, O’Day SJ, Sosman JA, Kirkwood JM, Eggermont AM, Dreno B, Nolop K, Li J, Nelson B, Hou J, Lee RJ, Flaherty KT, Mcarthur GA (2011) Improved survival with vemurafenib in melanoma with BRAF V600E mutation. $N$ Engl J Med 364: 2507-2516.

Chen B, Tardell C, Higgins B, Packman K, Boylan JF, Niu, H (2012) BRAFV600E negatively regulates the AKT pathway in melanoma cell lines. PLoS One 7: e42598.

Dale DC, Bolyard AA, Kelley ML, Westrup EC, Makaryan V, Aprikyan A, Wood B, Hsu FJ (2012) The CXCR4 antagonist plerixafor is a potential therapy for myelokathexis, WHIM syndrome. Blood 118: 4963-4966.

Davies H, Bignell GR, Cox C, Stephens P, Edkins S, Clegg S, Teague J, Woffendin H, Garnett MJ, Bottomley W, Davis N, Dicks E, Ewing R, Floyd Y, Gray K, Hall S, Hawes R, Hughes J, Kosmidou V, Menzies A, Mould C, Parker A, Stevens C, Watt S, Hooper S, Wilson R, Jayatilake H, Gusterson BA, Cooper C, Shipley J, Hargrave D, Pritchard-Jones K, Maitland N, Chenevix-Trench G, Riggins GJ, Bigner DD, Palmieri G, Cossu A, Flanagan A, Nicholson A, Ho JW, Leung SY, Yuen ST, Weber BL, Seigler HF, Darrow TL, Paterson H, Marais R, Marshall CJ, Wooster R, Stratton MR, Futreal PA (2002) Mutations of the BRAF gene in human cancer. Nature 417: 949-954.

de Snoo FA, Hayward NK (2005) Cutaneous melanoma susceptibility and progression genes. Cancer Lett 230: 153-186.

Dong J, Phelps RG, Qiao R, Yao S, Benard O, Ronai Z, Aaronson SA (2003) BRAF oncogenic mutations correlate with progression rather than initiation of human melanoma. Cancer Res 63: 3883-3885.

Franco R, Botti G, Mascolo M, Loquercio G, Liguori G, Ilardi G, Losito S, La Mura A, Calemma R, Ierano C, Bryce J, D’Alterio C, Scala S (2010) CXCR4-CXCL12 and VEGF correlate to uveal melanoma progression. Front Biosci (Elite Ed) 2: 13-21.

Gray-Schopfer V, Wellbrock C, Marais R (2007) Melanoma biology and new targeted therapy. Nature 445: 851-857.

Haughton EL, Tucker SJ, Marek CJ, Durward E, Leel V, Bascal Z, Monaghan T, Koruth M, Collie-Duguid E, Mann DA, Trim JE, Wright MC (2006) Pregnane X receptor activators inhibit human hepatic stellate cell transdifferentiation in vitro. Gastroenterology 131: 194-209.

Hong F, Tuyama A, Lee TF, Loke J, Agarwal R, Cheng X, Garg A, Fiel MI, Schwartz M, Walewski J, Branch A, Schecter AD, Bansal MB (2009) Hepatic stellate cells express functional CXCR4: role in stromal cellderived factor-1alpha-mediated stellate cell activation. Hepatology 49: 2055-2067.

Knall C, Worthen GS, Buhl AM, Johnson GL (1995) IL-8 signal transduction in human neutrophils. Ann N Y Acad Sci 766: 288-291.

Kuhnelt-Leddihn L, Muller H, Eisendle K, Zelger B, Weinlich G (2012) Overexpression of the chemokine receptors CXCR4, CCR7, CCR9, and CCR10 in human primary cutaneous melanoma: a potential prognostic value for CCR7 and CCR10? Arch Dermatol Res 304: 185-193.

Lovat PE, Corazzari M, Armstrong JL, Martin S, Pagliarini V, Hill D, Brown AM, Piacentini M, Birch-Machin MA, Redfern CP (2008) Increasing melanoma cell death using inhibitors of protein disulfide isomerases to abrogate survival responses to endoplasmic reticulum stress. Cancer Res 68: 5363-5369.

Mellor P, Harvey JR, Murphy KJ, Pye D, O’Boyle G, Lennard TW, Kirby JA, Ali S (2007) Modulatory effects of heparin and short-length oligosaccharides of heparin on the metastasis and growth of LMD MDA-MB 231 breast cancer cells in vivo. Br J Cancer 97: 761-768.

Mosi RM, Anastassova V, Cox J, Darkes MC, Idzan SR, Labrecque J, Lau G, Nelson K, Patel K, Santucci Z, Wong RSY, Skerlj RT, Bridger G, 
Huskens D, Schols D, Fricker SP (2012) The molecular pharmacology of AMD11070: an orally bioavailable CXCR4 HIV entry inhibitor. Biochem Pharmacol 83: 472-479.

Newton P, O’Boyle G, Jenkins Y, Ali S, Kirby JA (2009) T cell extravasation: demonstration of synergy between activation of CXCR3 and the T cell receptor. Mol Immunol 47: 485-492.

O'Boyle G, Fox CR, Walden HR, Willet JD, Mavin ER, Hine DW, Palmer JM, Barker CE, Lamb CA, Ali S, Kirby JA (2012) Chemokine receptor CXCR3 agonist prevents human T-cell migration in a humanized model of arthritic inflammation. Proc Natl Acad Sci USA 109: 4598-4603.

Schall TJ, Proudfoot AE (2011) Overcoming hurdles in developing successful drugs targeting chemokine receptors. Nat Rev Immunol 11: 355-363.

Smalley KS (2010) PLX-4032, a small-molecule B-Raf inhibitor for the potential treatment of malignant melanoma. Curr Opin Invest Drugs 11: 699-706.

Stone ND, Dunaway SB, Flexner C, Tierney C, Calandra GB, Becker S, Cao YJ, Wiggins IP, Conley J, Macfarland RT, Park JG, Lalama C, Snyder S, Kallungal B, Klingman KL, Hendrix CW (2007)

Multiple-dose escalation study of the safety, pharmacokinetics, and biologic activity of oral AMD070, a selective CXCR4 receptor inhibitor, in human subjects. Antimicrob Agents Chemother 51: 2351-2358.

Teicher BA, Fricker SP (2010) CXCL12 (SDF-1)/CXCR4 pathway in cancer. Clin Cancer Res 16: 2927-2931.

Thompson JA (2012) Ten years of progress in melanoma. J Natl Compr Cancer Netw 10: 932-935.

Yasumoto K, Koizumi K, Kawashima A, Saitoh Y, Arita Y, Shinohara K, Minami T, Nakayama T, Sakurai H, Takahashi Y, Yoshie O, Saiki I (2006) Role of the CXCl12/CXCR4 axis in peritoneal carcinomatosis of gastric cancer. Cancer Res 66: 2181-2187.

This work is published under the standard license to publish agreement. After 12 months the work will become freely available and the license terms will switch to a Creative Commons AttributionNonCommercial-Share Alike 3.0 Unported License. 